\title{
EQUIVALENT CONDITIONS TO THE SPECTRAL DECOMPOSITION PROPERTY FOR CLOSED OPERATORS
}

\author{
I. ERDELYI AND WANG SHENGWANG
}

\begin{abstract}
The spectral decomposition property has been instrumental in developing a local spectral theory for closed operators acting on a complex Banach space. This paper gives some necessary and sufficient conditions for a closed operator to possess the spectral decomposition property.
\end{abstract}

In the monograph [3] and in a sequel of papers by the authors, a local spectral theory has been built for closed operators on the sole assumption of the spectral decomposition property. As an abstraction of Dunford's concept of "spectral reduction' [2, p. 1927] and that of Bishop's "duality theory of type 3" [1], an operator $T$ endowed with the spectral decomposition property produces a spectral decomposition of the underlying space, pertinent to any finite open cover of the spectrum $\sigma(T)$. In this paper we obtain some conditions equivalent to the spectral decomposition property. Some of them generalize results from [4].

\section{Preliminaries}

Given a Banach space $X$ over the complex field $\mathbf{C}$, we denote by $C(X)$ the class of closed operators with domain $D_{T}$ and range in $X$, and we write $C_{d}(X)$ for the subclass of the densely defined operators in $C(X)$. For a subset $Y$ of $X, Y^{\perp}$ denotes the annihilator of $Y$ in $X^{*}$ and for $Z \subset X^{*}$, we use the symbol ${ }^{\perp} Z$ for the preannihilator of $Z$ in $X$. For the rest, the terminology and notation conform to that employed in [3].

We shall adopt and adjust some concepts and ideas from Bishop's "duality theory of type 4" [1, $\S 4]$. A couple $U_{1}$ and $U_{2}$ of a bounded and an unbounded Cauchy domain, related by $U_{2}=\left(\bar{U}_{1}\right)^{c}$, are referred to as complementary simple sets. $W_{1}$ and $W_{2}$ are the sets of analytic functions from $U_{1}$ to $X$ and from $U_{2}$ and $X^{*}$, respectively, which vanish at $\infty$. The seminorms

$$
\|f\|_{K_{1}}=\max \left\{\|f(\lambda)\|: f \in W_{1}, \lambda \in K_{1}, K_{1}\left(\subset U_{1}\right) \text { is compact }\right\}
$$

and

$$
\|g\|_{K_{2}}=\max \left\{\|g(\lambda)\|: g \in W_{2}, \lambda \in K_{2}, K_{2}\left(\subset U_{2}\right) \text { is compact }\right\}
$$

Received by the editors September 15, 1987.

1980 Mathematics Subject Classification (1985 Revision). Primary 47B40, Secondary 47A10, 47A15, 47A30. 
induce a locally convex topology on $W_{1}$ and $W_{2}$, respectively. For $i=1,2$ let $V_{i}$ be the subset of $W_{i}$ on which every function can be extended to be continuous on $\bar{U}_{i}$. For $f \in V_{1}, g \in V_{2}$, the norms

$$
\|f\|_{V_{1}}=\sup \left\{\|f(\lambda)\|: \lambda \in U_{1}\right\}, \quad\|g\|_{V_{2}}=\sup \left\{\|g(\lambda)\|: \lambda \in U_{2}\right\}
$$

make $\left(V_{1},\|\cdot\|_{V_{1}}\right)$ and $\left(V_{2},\|\cdot\|_{V_{2}}\right)$ Banach spaces. For $x \in X, \mu \in U_{1}$ and $\lambda \in U_{2}$, define

$$
\alpha(x, \mu, \lambda)=(\mu-\lambda)^{-1} x .
$$

For fixed $x \in X$ and $\lambda \in U_{2}, \alpha(x, \cdot, \lambda)$ is called an elementary element of $V_{1}$. Denote by $V$ the subspace of $V_{1}$ which is spanned by the elementary elements of $V_{1}$. For $x^{*} \in X^{*}, \mu \in U_{1}$ and $\lambda \in U_{2}$, define

$$
\alpha\left(x^{*}, \mu, \lambda\right)=(\mu-\lambda)^{-1} x^{*} \text {. }
$$

For fixed $x^{*} \in X^{*}$ and $\mu \in U_{1}$, call $\alpha\left(x^{*}, \mu, \cdot\right)$ and elementary element of $V_{2}$. For $f \in V_{1}$ and $g \in V_{2}$, with continuous extensions to $\Gamma=\partial U_{1}=$ $\partial U_{2}$, endow $\Gamma$ with the clockwise orientation and ascertain that the bilinear functional

$$
\Phi(f)=\langle f, g\rangle=\frac{1}{2 \pi i} \int_{\Gamma}\langle f(\lambda), g(\lambda)\rangle d \lambda
$$

is jointly continuous.

For $g \in V_{2},(1.3)$ defines a bounded linear functional $\Phi$ on $\dot{V}$, i.e. $\Phi \in V^{*}$. For $f=\alpha(x, \cdot, \lambda)$, one obtains

$$
\Phi(f)=\frac{1}{2 \pi i} \int_{\Gamma}(\mu-\lambda)^{-1}\langle x, g(\mu)\rangle d \mu=\langle x, g(\lambda)\rangle .
$$

The last equality holds because $g(\infty)=0$.

The proof of the following lemma is similar to that of [3, Lemma 9.1].

1.1. Lemma. Let $U_{1}, U_{2}$ be complementary simple sets. With $V, V_{i}$ and $W_{i}$ $(i=1,2)$, as defined above, there exists a linear manifold $Y$ in $W_{2}$ and a norm on $Y$ such that

(i) $Y$ is a Banach space isometrically isomorphic to $V^{*}$ :

(ii) $V_{2} \subset Y$;

(iii) the mappings $V_{2} \rightarrow Y$ and $Y \rightarrow W_{2}$ are continuous;

(iv) the inner product between $V$ and $V_{2}$, defined by (1.3), can be extended to an inner product between $V$ and $Y$ in conjunction with the isometric isomorphism between $Y$ and $V^{*}$, as asserted by (i).

\section{SOME DUAL PROPERTIES}

For an operator $T \in C_{d}(X)$, define an operator $H$ on $V$ by

$$
D_{H}=\{f \in V: T f(\mu) \in V\}, \quad(H f)(\mu)=(\mu-T) f(\mu) .
$$


2.1. Lemma. The operator $H$ is closed and densely defined on $V$.

Proof. For $f=\alpha(x, \cdot, \lambda)$ with $x \in D_{T}$, one has $f \in D_{H}$ and

$$
(H f)(\mu)=(\mu-\lambda)^{-1}(\mu-T) x=x+(\mu-\lambda)^{-1}(\lambda-T) x .
$$

The linear span of all elementary elements being dense in $V$, the operator $H$ is densely defined.

Let $\left\{f_{n}\right\}$ be a sequence in $D_{H}$ such that $f_{n} \rightarrow f$ and $H f_{n} \rightarrow g$, for some functions $f$ and $g$. T being closed, it follows from

$$
\left(H f_{n}\right)(\mu)=(\mu-T) f_{n}(\mu) \text {, }
$$

that $f \in D_{H}$ and

$$
(H f)(\mu)=(\mu-T) f(\mu)=g(\mu), \quad \mu \in \bar{U}_{1} .
$$

Thus $H$ is closed.

The next lemma defines the dual $H^{*}$ of $H$. Henceforth, $g$ will denote a typical element of $V^{*}=Y$.

2.2. Lemma. The dual operator of $H$ is defined by

$$
\left(H^{*} g\right)(\lambda)=-\lim _{\lambda \rightarrow \infty} \lambda g(\lambda)+\left(\lambda-T^{*}\right) g(\lambda), \quad g \in D_{H^{*}}
$$

Proof. For $f(\mu)=\alpha(x, \mu, \lambda)$ with $x \in D_{T}$ and $\lambda \in U_{2}$ fixed, and $g \in D_{H^{*}}$, (2.1), (1.3) and (1.4) imply

$$
\begin{aligned}
\left\langle f, H^{*} g\right\rangle & =\langle H f, g\rangle=\langle x, g\rangle+\langle\alpha((\lambda-T) x, \cdot, \lambda), g\rangle \\
& =\langle x, g\rangle+\langle(\lambda-T) x, g(\lambda)\rangle,
\end{aligned}
$$

where $x$, as a function of $\mu \in \bar{U}_{1}$, is an element of $V_{1}$. It follows from

$$
x=\frac{1}{2 \pi i} \int_{\Gamma^{\prime}}(\mu-\lambda)^{-1} x d \lambda=\frac{1}{2 \pi i} \int_{\Gamma^{\prime}} \alpha(x, \mu, \lambda) d \lambda,
$$

that $x \in V$, where $\Gamma^{\prime}$ is a closed $C^{2}$-Jordan curve with the clockwise orientation that contains $\Gamma$ in its interior. Furthermore, with the help of (1.4), one obtains

$$
\begin{aligned}
\langle x, g\rangle & =\frac{1}{2 \pi i} \int_{\Gamma^{\prime}}\langle\alpha(x, \cdot, \lambda), g\rangle d \lambda=\frac{1}{2 \pi i} \int_{\Gamma^{\prime}}\langle x, g(\lambda)\rangle d \lambda \\
& =\lim _{z \rightarrow \infty} z\left(-\frac{1}{2 \pi i} \int_{\Gamma^{\prime}}(\lambda-z)^{-1}\langle x, g(\lambda)\rangle d \lambda\right)=-\lim _{z \rightarrow \infty} z\langle x, g(z)\rangle \\
& =-\lim _{\lambda \rightarrow \infty} \lambda\langle x, g(\lambda)\rangle=\left\langle x,-\lim _{\lambda \rightarrow \infty} \lambda g(\lambda)\right\rangle .
\end{aligned}
$$

It follows from (2.3) and (2.4) that

$$
\begin{aligned}
\left\langle f, H^{*} g\right\rangle & =\left\langle x,-\lim _{\lambda \rightarrow \infty} \lambda g(\lambda)\right\rangle+\langle(\lambda-T) x, g(\lambda)\rangle \\
& =\left\langle x,-\lim _{\lambda \rightarrow \infty} \lambda g(\lambda)+(\lambda-T)^{*} g(\lambda)\right\rangle .
\end{aligned}
$$


In fact, $f=\alpha(x, \cdot, \lambda)$ and since $\left\langle f, H^{*} g\right\rangle$ and $\left\langle x,-\lim _{\lambda \rightarrow \infty} \lambda g(\lambda)\right\rangle$ are bounded linear functionals of $x$, so is $\langle(\lambda-T) x, g(\lambda)\rangle$. Thus $g(\lambda) \in D_{T^{*}}$, for every $\lambda \in U_{2}$ and hence the last equality of (2.5) holds. Now (2.5) combined with (1.3) and (1.4), gives $\left\langle f, H^{*} g\right\rangle=\left\langle x,\left(H^{*} g\right)(\lambda)\right\rangle$ and hence $H^{*}$ is expressed by (2.2).

Define the map $\tau: V^{*} \rightarrow X^{*}$ by $\tau g=\lim _{\lambda \rightarrow \infty} \lambda g(\lambda)$. Then $H^{*}$, given by (2.2), is expressed by

$$
\left(H^{*} g\right)(\lambda)=-\tau g+\left(\lambda-T^{*}\right) g(\lambda) \text {. }
$$

2.3. Lemma. Let $x^{*} \in X^{*}$. Then $x^{*} \in D_{T^{*}}$ iff there exists $g \in D_{H^{*}}$ such that $\tau g=x^{*}$.

Proof. First, assume that there is $g \in D_{H^{*}}$ such that $\tau g=x^{*}$. Since $H^{*} g \in$ $V^{*}$, the following limit exists

$$
\lim _{\lambda \rightarrow \infty} T^{*} \lambda g(\lambda)=\lim _{\lambda \rightarrow \infty} \lambda T^{*} g(\lambda) \text {. }
$$

Furthermore, $\lim _{\lambda \rightarrow \infty} \lambda g(\lambda)$ also exists and since $T$ is closed, we have

$$
x^{*}=\tau g=\lim _{\lambda \rightarrow \infty} \lambda g(\lambda) \in D_{T^{*}} .
$$

Conversely, for every $x^{*} \in D_{T^{*}}$, the corresponding elementary element $\alpha\left(x^{*}, \mu, \cdot\right)$ with $\mu \in U_{1}$ fixed, is in $D_{H^{*}}$. It follows from (1.2), that

$$
\tau(-\alpha)=-\lim \lambda \alpha\left(x^{*}, \mu, \lambda\right)=x^{*}
$$

and the proof reaches its conclusion by setting $g=-\alpha$.

\section{NORMS ON THE DUAL SPACES}

We introduce the norm

$$
\left\|\left(f_{1}, f_{2}\right)\right\|_{\eta}=\left(\eta\left\|f_{1}\right\|^{2}+\left\|f_{2}\right\|^{2}\right)^{1 / 2}, \quad \eta>0,
$$

in $V \oplus V$. This induces the norm

$$
\left\|\left(g_{1}, g_{2}\right)\right\|_{\eta}=\left(\eta^{-1}\left\|g_{1}\right\|^{2}+\left\|g_{2}\right\|^{2}\right)^{1 / 2}
$$

in $V^{*} \oplus V^{*}$. Let $G(H)$ and $G\left(H^{*}\right)$ be the graphs of $H$ and $H^{*}$, respectively. $G(H)$, as a subspace of $V \oplus V$, is endowed with the norm

$$
\|(f, H f)\|_{\eta}=\left(\eta\|f\|^{2}+\|H f\|^{2}\right)^{1 / 2} .
$$

It follows from

$$
(G(H))^{\perp}=\nu G\left(H^{*}\right), \quad \text { where } \nu\left(g_{1}, g_{2}\right)=\left(-g_{2}, g_{1}\right),
$$

that $\nu G\left(H^{*}\right)$ is the dual of $(V \oplus V) / G(H)$. The latter is equipped with the norm

$$
\left\|\left(f_{1}, f_{2}\right)^{-}\right\|_{\eta}=\inf \left\{\left(\left\|f_{1}-f\right\|^{2}+\left\|f_{2}-H f\right\|^{2}\right)^{1 / 2}: f \in D_{H}\right\}
$$


where $\left(f_{1}, f_{2}\right)$ denotes a typical element of $(V \oplus V) / G(H)$. To the norm (3.2), there corresponds the following norm in $\nu G\left(H^{*}\right)$ :

$$
\left\|\left(-H^{*} g, g\right)\right\|_{\eta}=\left(\eta^{-1}\left\|H^{*} g\right\|^{2}+\|g\|^{2}\right)^{1 / 2} .
$$

\subsection{Lemma. The norm}

$$
\left\|x^{*}\right\|_{T^{*}}=\left(\left\|x^{*}\right\|^{2}+\left\|T x^{*}\right\|^{2}\right)^{1 / 2}
$$

in $D_{T^{*}}$ is equivalent to the norm

$$
\left\|x^{*}\right\|_{\eta}=\inf \left\{\left(\eta^{-1}\left\|H^{*} g\right\|^{2}+\|g\|^{2}\right)^{1 / 2}: \tau g=x^{*}\right\} .
$$

Furthermore, $D_{T^{*}}$ equipped with the norm (3.3) or (3.4) is the dual of a Banach space.

Proof. First, we prove that $D_{T^{*}}$ endowed with the norm (3.4) is a Banach space. Let $\left\{x_{n}^{*}\right\}$ be a Cauchy sequence with respect to the norm (3.4). Without loss of generality, we may suppose that

$$
\sum_{n=0}^{\infty}\left\|x_{n+1}^{*}-x_{n}^{*}\right\|_{\eta}<\infty, \quad x_{0}=0 .
$$

For each $x_{n}$, we may choose $g_{n} \in D_{H^{*}}$ such that

$$
\left.\left(\eta^{-1}\left\|H^{*}\left(g_{n+1}-g_{n}\right)\right\|^{2}+\| g_{n+1}-g_{n}\right) \|^{2}\right)^{1 / 2} \leq 2\left\|x_{n+1}^{*}-x_{n}^{*}\right\|_{\eta}
$$

and $\tau g_{n}=x_{n}^{*}$. Relations (3.5) and (3.6) imply that both $\left\{g_{n}\right\}$ and $\left\{H^{*} g_{n}\right\}$ converge. Put $g=\lim _{n \rightarrow \infty} g_{n}$. $H^{*}$ being closed, one has $g \in D_{H^{*}}$ and $H^{*} g=\lim _{n \rightarrow \infty} H^{*} g_{n}$. Then Lemma 2.3 implies that $x^{*}=\tau g \in D_{T^{*}}$. Since

$$
\left\|x_{n}^{*}-x^{*}\right\|_{\eta} \leq\left(\eta^{-1}\left\|H^{*}\left(g_{n}-g\right)\right\|^{2}+\left\|g_{n}-g\right\|^{2}\right)^{1 / 2} \rightarrow 0 \quad n \rightarrow \infty,
$$

it follows that $D_{T^{*}}$, endowed with the norm (3.4), is a Banach space.

To show that the norms (3.3) and (3.4) are equivalent, let $x^{*} \in D_{T^{*}}$ and $g=\alpha\left(x^{*}, \mu, \cdot\right)$ with $\mu \in U_{1}$ fixed. Since $\tau g=x^{*}$, one has

$$
\begin{aligned}
\left\|x^{*}\right\|_{\eta} & \leq\left(\eta^{-1}\left\|H^{*} g\right\|^{2}+\|g\|^{2}\right)^{1 / 2} \\
& \leq \frac{1}{\delta}\left(\eta^{-1}\left(|\mu| \cdot\left\|x^{*}\right\|+\left\|T^{*} x^{*}\right\|\right)^{2}+\left\|x^{*}\right\|^{2}\right)^{1 / 2},
\end{aligned}
$$

where $\delta=\operatorname{dist}\left(\mu, U_{2}\right)$. In view of (3.7), there exists $K_{\eta}>0$ such that

$$
\left\|x^{*}\right\|_{\eta} \leq K_{\eta}\left(\left\|x^{*}\right\|^{2}+\left\|T^{*} x^{*}\right\|^{2}\right)^{1 / 2}=K_{\eta}\left\|x^{*}\right\|_{T^{*}} .
$$

$D_{T^{*}}$ being complete with respect to both $\|\cdot\|_{\eta}$ and $\|\cdot\|_{T^{*}},(3.8)$ implies that the two norms are equivalent. $D_{T}$. equipped with the norm (3.3) is isometrically isomorphic to $\nu G\left(T^{*}\right) \quad\left(=G(T)^{\perp}\right)$. Since $\nu G\left(T^{*}\right)$ is the dual of $X \oplus X / G(T)$, so is $D_{T^{*}}$.

$D_{T^{*}}$ equipped with either of the two norms (3.3), (3.4), will be denoted by $D$. To obtain a further property of $\tau$, we need the following. 
3.2. Lemma. Let $Y, Z$ be Banach spaces and let $S$ be a bounded surjective map of $Y$ onto $Z$. In $Z$ we define the norm

$$
\|z\|_{S}=\inf \{\|y\|: y \in Y, S y=z\}, \quad z \in Z \text {. }
$$

Then, the corresponding norm in the dual space $Z^{*}$ is

$$
\left\|z^{*}\right\|_{S^{*}}=\left\|S^{*} z^{*}\right\|, \quad z^{*} \in Z^{*} .
$$

Proof. Let $N(S)$ be the null space of $S$. Then $N(S)^{\perp}$ is the dual of $Y / N(S)$. Let $y_{0} \in Y, z=S y_{0}$ and let $\hat{y}_{0}$ be the equivalence class of $y_{0}$ in $Y / N(S)$. In terms of the norm (3.9), one has

$$
\left\|\hat{y}_{0}\right\|=\inf \left\{\left\|y_{0}-w\right\|: w \in N(S)\right\}=\inf \{\|y\|: S y=z\}=\|z\|_{S} .
$$

The dual norm of $\left\|\hat{y}_{0}\right\|$ in $N(S)^{\perp}$ is the usual norm in $Y^{*}$, restricted to $N(S)^{\perp}$. Note that $S^{*}$ is a surjective map from $Z^{*}$ onto $N(S)^{\perp}$. Therefore, the corresponding norm of $\|\cdot\|_{S}$ in $Z^{*}$ is the one expressed by (3.10).

We define an operator $K$ from $\nu G\left(H^{*}\right)$ into $D_{T^{*}}$ by $K\left(-H^{*} g, g\right)=\tau g$. Let $D^{\#}$ be the dual of $D$. Then $K^{\#}$, the dual of $K$, is an operator from $D^{\#}$ into the dual of $\nu G\left(H^{*}\right)$, i.e. from $D^{\#}$ into $\left(V^{* *} \oplus V^{* *}\right) /\left(\nu G\left(H^{*}\right)\right)^{\perp}$.

For every $x \in X$, define a continuous linear functional $\psi$ on $D$, by

$$
\psi\left(x^{*}\right)=\left\langle x, x^{*}\right\rangle, \quad x^{*} \in D .
$$

3.3. Lemma. The linear functional $\psi(3.11)$ is a zero functional only if $x=0$. Proof. Assume that $\psi=0$. Then $\left\langle x, x^{*}\right\rangle=0$ for every $x^{*} \in D$. Thus, we have $\left\langle 0,-T^{*} x^{*}\right\rangle+\left\langle x, x^{*}\right\rangle=0, x^{*} \in D$, equivalently, $(0, x) \perp \nu G\left(T^{*}\right)$, i.e. $(0, x) \in G(T)$. Consequently, $x=0$.

In view of Lemma 3.3, we may consider $X$ as a subset of $D^{\#}$. In the following, we shall have a closer look at $K^{\#} x$ for $x \in X$.

For $x^{*} \in D$ and fixed $\mu \in U_{1}$, put $g=\alpha\left(x^{*}, \mu, \cdot\right)$. Then, one obtains

$$
\begin{aligned}
\left\langle K^{*} x\left(-H^{*} g, g\right)\right\rangle & =\left\langle x, K\left(-H^{*} g, g\right)\right\rangle=\langle x, \tau g\rangle=\left\langle x, x^{*}\right\rangle \\
& =\frac{1}{2 \pi i} \int_{\Gamma}(\mu-\lambda)^{-1}\left\langle x, x^{*}\right\rangle d \lambda=\langle x, g\rangle,
\end{aligned}
$$

where $\Gamma$ has a clockwise orientation.

We know that $(0, x)$ is an element of $X \oplus X$. We may also consider $(0, x)$ as an element of $V \oplus V$ and hence $(0, x)$ can be assumed to be an element of $V^{* *} \oplus V^{* *}$.

Thus, we have

$$
\langle x, g\rangle=\left\langle(0, x),\left(-H^{*} g, g\right)\right\rangle=\left\langle(0, x)^{\sim},\left(-H^{*} g, g\right)\right\rangle,
$$

where $(0, x)^{\sim}$ is the equivalence class of $(0, x)$ in $\left(V^{* *} \oplus V^{* *}\right) /\left(\nu G\left(H^{*}\right)\right)^{\perp}$. Consequently, $K^{\#} x=(0, x)^{\sim}$.

Denote by $(0, x)^{-}$the equivalence class of $(0, x)$ in $(V \oplus V) / G(H)$. 
3.4. Lemma. Let $J$ be the natural embedding of $(V \oplus V) / G(H)$ into

$$
\left(V^{* *} \oplus V^{* *}\right) /\left(\nu G\left(H^{*}\right)\right)^{\perp} \text {. }
$$

Then $J(0, x)^{\wedge}=(0, x)^{\sim}$.

Proof. For any $\left(-H^{*} g, g\right) \in \nu G\left(H^{*}\right)$, one has

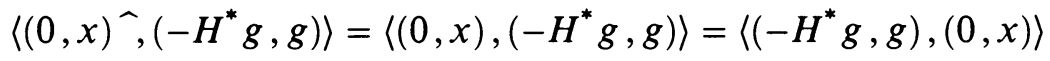

$$
\begin{aligned}
& =\left\langle\left(-\left(H^{*} g, g\right),(0, x)^{\sim}\right\rangle .\right.
\end{aligned}
$$

Note that while in $\left\langle(0, x),\left(-H^{*} g, g\right)\right\rangle,(0, x) \in V \oplus V$; in $\left\langle\left(-H^{*} g, g\right)\right.$, $(0, x)\rangle,(0, x)$ is considered an element of $V^{* *} \oplus V^{* *}$. It follows from the above equalities that $J(0, x)^{\wedge}=(0, x)^{\sim}$.

In particular, Lemma 3.4 implies

$$
\left\|(0, x)^{\wedge}\right\|=\left\|(0, x)^{\sim}\right\| .
$$

On the other hand, $(0, x)^{\wedge}=0$ implies $(0, x) \in G(H)$ and the latter implies $x=0$. Accordingly, we may define the following norm on $X$ :

$$
\|x\|_{\eta}=\left\|(0, x)^{\wedge}\right\|=\inf \left\{\left(\eta\|f\|^{2}+\|x-H f\|^{2}\right)^{1 / 2}: f \in D_{H}\right\} .
$$

In view of Lemma 3.4, we may consider $K^{\#} x=(0, x)^{\sim}$ as a point of $(V \oplus V) / G(H)$.

3.5. Lemma. The norm $\|\cdot\|_{\eta}$, defined by (3.13), is the restriction of the norm on $D^{\#}$.

Proof. The space $\left(V^{* *} \oplus V^{* *}\right) /\left(\nu G\left(H^{*}\right)\right)^{\perp}$ is the conjugate of $\nu G\left(H^{*}\right)$ and $K$ is an operator from $\nu G\left(H^{*}\right)$ into $D$. It follows from Lemma 3.2, that the dual norm on $D^{\#}$ is given by

$$
\left\|x^{\#}\right\|=\left\|K^{\#} x^{\#}\right\|_{* *}
$$

where $x^{*} \in D^{*}$ and $\|\cdot\|_{* *}$ is the norm on $V^{* *} \oplus V^{* *} /\left[\nu G\left(H^{*}\right)\right]^{\perp}$. If $x^{\#}=$ $x \in X$, then the norm (3.14) becomes $\|x\|_{\eta}=\left\|K^{\#} x\right\|=\left\|(0, x)^{\sim}\right\|$ and it follows from (3.12) that the restriction of the norm (3.14) to $X$ is that given by (3.13).

\section{A DUALITY PROPERTY OF SOME SPECTRAL-TYPE MANIFOLDS}

Define the following linear manifolds in $X$ :

$$
\begin{aligned}
& N=\left\{x \in X: \text { for every } \varepsilon>0, \text { there exists } f \in D_{H} \text { with }\|x-H f\|<\varepsilon\right\}, \\
& M=\left\{x^{*} \in D \text { : there exists } g \in D_{H^{*}} \text { such that } H^{*} g=0, \tau g=x^{*}\right\} .
\end{aligned}
$$

4.1. Lemma. The manifolds $N$ and $M$ have the following characterizations:

$$
\begin{aligned}
& N=\left\{x \in X:\|x\|_{\eta} \rightarrow 0 \text { as } \eta \rightarrow 0\right\}, \\
& M=\left\{x^{*} \in D:\left\|x^{*}\right\|_{\eta} \leq R \text { for } n>0 \text { and } R \text { depends on } x^{*}\right\} .
\end{aligned}
$$


Proof. First, we establish (4.1). Let $x \in N$. Since, for every $\varepsilon>0$, there is $f \in D_{H}$ such that $\|x-H f\|<\varepsilon$, it follows from (3.13) that $\overline{\lim }_{n \rightarrow 0}\|x\|_{\eta} \leq \varepsilon$. $\varepsilon$ being arbitrary, it follows that $\lim _{\eta \rightarrow 0}\|x\|_{\eta}=0$.

Conversely, suppose that $\|x\|_{\eta} \rightarrow 0$ as $\eta \rightarrow 0$. Then, for every $\varepsilon>0$, there exists $\eta>0$ such that $\|x\|_{\eta}<\varepsilon$ and hence $\|x-H f\|<\varepsilon$ for some $f \in D_{H}$.

Next, we prove (4.2). It is a straightforward consequence of (3.4) that

$$
M \subset\left\{x^{*} \in D:\left\|x^{*}\right\|_{\eta} \text { is bounded for } \eta>0\right\} \text {. }
$$

Conversely, suppose that $x^{*} \in D$ and $\left\|x^{*}\right\|_{\eta}$ is bounded for $\eta>0$, i.e. there exists $R>0$ such that

$$
\inf \left\{\left(n\left\|H^{*} g\right\|^{2}+\|g\|^{2}\right)^{1 / 2}, \tau g=x^{*}\right\}<R, \quad n=1,2, \ldots
$$

Then, for every $n$, there exists $g_{n} \in D_{H^{*}}$ satisfying conditions

$$
n\left\|H^{*} g_{n}\right\|^{2}+\left\|g_{n}\right\|^{2} \leq R^{2} \text { and } \tau g_{n}=x^{*} \text {. }
$$

In view of (4.3), the sequences $\left\{g_{n}\right\}$ and $\left\{H^{*} g_{n}\right\}$ are bounded and hence the sequence $\left\{\left(H^{*} g_{n}, g_{n}\right)\right\}$ is bounded. Consequently, $\left\{\left(-H^{*} g_{n}, g_{n}\right)\right\}$ has a cluster point $(h, g)$ in $V^{*} \oplus V^{*}$, with respect to the weak* topology of $V^{*} \oplus V^{*}$. Since $\nu G\left(H^{*}\right)$ is closed with respect to the same topology, one has $\{h, g\} \in \nu G\left(H^{*}\right)$, i.e. $h=-H^{*} g$. It follows from $\left\|H^{*} g_{n}\right\| \leq R^{2} / n$ that $\left\|H^{*} g\right\|=0$.

On the other hand, for every $x \in X, K^{\#} x=(0, x)^{\sim} \in(V \oplus V) / G(H)$. Therefore,

$$
\left\langle x, x^{*}\right\rangle=\left\langle x, \tau g_{n}\right\rangle=\left\langle x, K\left(-H^{*} g_{n}, g_{n}\right)\right\rangle=\left\langle K^{\#} x,\left(-H^{*} g_{n}, g_{n}\right)\right\rangle
$$

Since $\left(-H^{*} g, g\right)$ is also a cluster point of $\left\{\left(-H^{*} g_{n}, g_{n}\right)\right\}$ in the weak* topology of $\nu G\left(H^{*}\right)$, the latter being the dual space of $(V \oplus V) / G(H)$, we have

$$
\left\langle x, x^{*}\right\rangle=\left\langle K^{\#} x,\left(-H^{*} g, g\right)\right\rangle=\left\langle x, K\left(-H^{*} g, g\right)\right\rangle=\langle x, \tau g\rangle \text {. }
$$

Thus $\tau g=x^{*}$ and hence $x^{*} \in M$. Expression (4.2) is obtained.

4.2. Theorem. $N$ and $M$, as defined above, are related by

$$
N^{\perp}=\bar{M}^{w},
$$

where $^{w}$ denotes the weak ${ }^{*}$ closure in $X^{*}$.

Proof. Let $x \in N$ and $x^{*} \in M$. It follows from Lemmas 3.5 and 4.1, that

$$
\left|\left\langle x, x^{*}\right\rangle\right| \leq\|x\|_{\eta} \cdot\left\|x^{*}\right\|_{\eta} \rightarrow 0 \quad(\text { as } \eta \rightarrow 0) .
$$

Therefore, $N^{\perp} \supset \bar{M}^{w}$.

Next, we prove the opposite inclusion. For $x \notin N(x \in X)$, Lemma 4.1 implies that there exists $\eta_{n} \downarrow 0$ such that

$$
\|x\|_{\eta_{n}}>C>0
$$

for some constant $C$. In view of (4.4), we can find $x_{n}^{*} \in D$ such that $\left\|x_{n}^{*}\right\|_{\eta_{n}} \leq 1$ and $\left|\left\langle x, x_{n}^{*}\right\rangle\right|>C$. The sequence $\left\{\eta_{n}\right\}$ being nonincreasing, so is the norm 
(3.4), i.e. $\left\|x_{n}^{*}\right\|_{\eta_{1}} \leq\left\|x_{n}^{*}\right\|_{\eta_{n}}$. Consequently, $\left\{x_{n}^{*}\right\}$ is bounded in the norm $\|\cdot\|_{\eta_{1}}$-topology. For every $n$, there exists $g_{n} \in D_{H^{*}}$ such that

$$
\eta_{n}^{-1}\left\|H^{*} g_{n}\right\|^{2}+\left\|g_{n}\right\|^{2} \leq 2\left\|x_{n}^{*}\right\|_{\eta_{n}}^{2}
$$

Thus $\left\{\left(x_{n}^{*}, g_{n}, H^{*} g_{n}\right)\right\}$ is bounded in $D \oplus G\left(H^{*}\right)$. By Lemma 3.1 and the previous paragraph, $D \oplus G\left(H^{*}\right)$ is the dual of a Banach space and $\left\{\left(x_{n}^{*}, g_{n}, H^{*} g_{n}\right)\right\}$ has a cluster point $\left(x^{*}, g, H^{*} g\right)$ in the weak* topology of $D \oplus G\left(H^{*}\right)$. Since (3.11) defines a continuous linear functional on $D$ for every $x \in X$, it follows that $x^{*}$ is also a cluster point of $\left\{x_{n}^{*}\right\}$ in the weak* topology of $X^{*}$. Now it follows from the inequalities

$$
\left\langle x, x_{n}^{*}\right\rangle=\left\langle x, \tau g_{n}\right\rangle=\left\langle x, K\left(-H^{*} g_{n}, g_{n}\right)\right\rangle=\left\langle K^{*} x,\left(-H^{*} g_{n}, g_{n}\right)\right\rangle
$$

that, for $x \in X$, one has

$$
\left\langle x, x^{*}\right\rangle=\left\langle K^{*} x,\left(-H^{*} g, g\right)\right\rangle=\left\langle x, K\left(-H^{*} g, g\right)\right\rangle=\langle x, \tau g\rangle .
$$

Thus $x^{*}=\tau g$. By the definition of $M, x^{*} \in M$. Hence $N \supset^{\perp} M$, or equivalently, $N^{\perp} \subset \bar{M}^{w}$.

\section{THE MAIN THEOREM}

We recall the definition of the central topic of this paper.

5.1. Definition. An operator $T \in C(X)$ is said to have the spectral decomposition property (SDP) if, for every finite open cover $\left\{G_{i}\right\}_{i=0}^{n}$ of $C($ or $\sigma(T))$, where $G_{0}$ is a neighborhood of infinity (i.e. its complement $G_{0}^{c}$ is compact in $\mathrm{C}$ ), there exists a system $\left\{Y_{i}\right\}_{i=0}^{n}$ of invariant subspaces under $T$ satisfying the following conditions:

(I) $X_{i} \subset D_{T}$ if $G_{i}(1 \leq i \leq n)$ is relatively compact;

(II) $\sigma\left(T \mid X_{i}\right) \subset G_{i} \quad(0 \leq i \leq n)$;

(III) $X=\sum_{i=0}^{n} X_{i}$.

The theory based on this property is greatly simplified by the fact [3, Corollary 6.3] that $T$ has the SDP iff it has the two-summand spectral decomposition property that corresponds to $n=1$. The theory also involves the concept of the spectral manifold $X(T, H)=\{x \in X: \sigma(x, T) \subset H\}$, where $H \subset \mathrm{C}$ and $\sigma(x, T)$ is the local spectrum at $x \in X$, and the concept of the $T$-bounded spectral maximal space $\Xi(T, F)$ for $F \subset C$ compact. The $T$-bounded spectral maximal space $\Xi(T, F)$ is associated to $X(T, F)$ [3, Theorem 4.34] by

$$
X(T, F)=\Xi(T, F) \oplus X(T, \varnothing) \text { and } \sigma(T \mid \Xi(T, F))=\sigma(T \mid X(T, F)) .
$$

The given operator $T$ may enjoy two specific properties:

$T$ is said to have property $(\beta)$ [1, Definition 8 and 3, Definition 5.5] if, for any sequence $\left\{f_{n}: G \rightarrow D_{T}\right\}$ of analytic functions, the condition $(\lambda-T) f_{n}(\lambda) \rightarrow$ 0 (as $n \rightarrow \infty$ ) in the strong topology of $X$ and uniformly on every compact 
subset of $G$ implies that $f_{n}(\lambda) \rightarrow 0$ in the strong topology of $X$ and uniformly on every compact subset of $G$.

$T$ is said to have property $(\kappa)$ [3, Definition 5.4] if $T$ has the single valued extension property and $X(T, F)$ is closed for every closed $F$.

Property $(\beta)$ implies property $(\kappa)$, as follows from [3, Proposition 5.6].

5.2. Lemma. Suppose that $S \in C\left(X^{*}\right)$. Then $S$ is the dual of a closed and densely defined operator $T \in C_{d}(X)$ iff $G(S)$ is closed in the weak ${ }^{*}$ topology of $X^{*} \oplus X^{*}$ and $D_{S}$ is total.

Proof. Only if: Suppose that $S$ is the dual of $T \in C_{d}(X)$, i.e. $S=T^{*}$. The equality

$$
\nu G(S)=\nu G\left(T^{*}\right)=(G(T))^{\perp}
$$

implies $G(S)$ is closed in the weak* topology of $X^{*} \oplus X^{*}$. To prove that $D_{S}$ is total, let $x \in X$ and $\left\langle x, x^{*}\right\rangle=0$ for all $x^{*} \in D_{S}$. Then

$$
\left\langle x, x^{*}\right\rangle=0=\left\langle 0, S x^{*}\right\rangle
$$

is equivalent to

$$
0 \oplus x \in^{\perp}(\nu G(s))=G(T)
$$

and hence $x=T(0)=0$, so $D_{S}$ is total.

If: Assume that $G(S)$ is closed in the weak* topology of $X^{*} \oplus X^{*}$ and $D_{S}$ is total. Letting $W={ }^{\perp}(\nu G(S))$, one has $W={ }^{\perp} \nu G(S)$. Let $0 \oplus y \in W$. For every $x^{*} \in D_{S}$, one has $0 \oplus y \perp\left(-S x^{*}\right) \oplus x^{*}$, or equivalently,

$$
0=\left\langle 0, S x^{*}\right\rangle=\left\langle y, x^{*}\right\rangle \text { for all } x^{*} \in D_{s} .
$$

$D_{S}$ being total, (5.1) implies that $y=0$ and hence $W$ is the graph of an operator $T$. $W$ being closed, $T$ is a closed operator.

To show that $T$ is densely defined, let $x^{*} \in X^{*}$ satisfy condition

$$
\left\langle x, x^{*}\right\rangle=0 \text { for all } x \in D_{T} \text {. }
$$

Then

$$
x \oplus T x \perp x^{*} \oplus 0 \text { for all } x \in D_{T}
$$

and hence $x^{*} \oplus 0 \in(G(T))^{\perp}=W^{\perp}=\nu G(S)$. Therefore $x^{*}=-S(0)=0$ and hence $T$ is densely defined.

5.3. Lemma. Suppose that $T \in C(X)$ and $Y$ is invariant under $T$. Then $T / Y$ is closed iff $G(T / Y)$ is topologically isomorphic to $G(T) / G(T \mid Y)$.

Proof. Only if: Assume that $T / Y$ is closed. For $x \in D_{T}$, the following mapping $x \oplus T x+G(T \mid Y) \rightarrow(x \oplus Y)+(T x+y)$ is bijective from $G(T) / G(T \mid Y)$ onto $G(T / Y)$. It follows from the inequalities

$$
\begin{aligned}
\|x \oplus T x+G(T \mid Y)\| & =\inf \left\{\|x \oplus T x+y \oplus T y\|: y \in D_{T \mid Y}\right\} \\
& \geq \inf \left\{\left\|\left(x+y_{1}\right) \oplus\left(T x+y_{2}\right)\right\|: y_{1}, y_{2}, \in Y\right\} \\
& =\|(x+Y) \oplus(T x+Y)\|
\end{aligned}
$$


and from the open mapping theorem that $G(T / Y)$ and $G(T) / G(T \mid Y)$ are topologically isomorphic.

If: Assume that $G(T / Y)$ and $G(T) / G(T \mid Y)$ are topologically isomorphic. Then $G(T / Y)$ is a Banach space and hence it is closed in $X / Y \oplus X / Y$. Thus $T / Y$ is closed.

5.4. Lemma. Given $T \in C_{d}(X)$, let $Z \subset D_{T}$ be an invariant subspace under T. Then

(i) $Z^{\perp}$ is invariant under $T^{*}$;

(ii) $T^{*} / Z^{\perp}$ is the dual of $T \mid Z$ iff $T^{*} / Z^{\perp}$ is closed.

Proof. (i) is evident.

(ii): If $T^{*} / Z^{\perp}$ is the dual of $T \mid Z$ then clearly $T^{*} / Z^{\perp}$ is closed. Conversely, assume that $T^{*} / Z^{\perp}$ is closed. Then, it follows from Lemma 5.3 that $G\left(T^{*} / Z^{\perp}\right)$ is topologically isomorphic to $G\left(T^{*}\right) / G\left(T^{*} \mid Z^{\perp}\right)$. The following equalities

$$
\nu G\left(T^{*}\right)=(G(T))^{\perp} ; \quad G\left(T^{*} \mid Z^{\perp}\right)=G\left(T^{*}\right) \cap\left(Z^{\perp} \oplus Z^{\perp}\right)
$$

imply that both $G\left(T^{*}\right)$ and $G\left(T^{*} \mid Z^{\perp}\right)$ are closed in the weak* topology of $X^{*} \oplus X^{*}$. Then, it follows easily that $G\left(T^{*} / Z^{\perp}\right)$ is closed in the weak* topology of $X^{*} / Z^{\perp} \oplus X^{*} / Z^{\perp}$.

It follows from Lemma 5.2 that $D_{T^{*}}$ is total and hence $D_{T^{*} / Z^{\perp}}$ is total. Quoting again Lemma 5.2, it follows that $T^{*} / Z^{\perp}$ is the dual of a densely defined closed operator $U \in C_{d}(Z)$.

The assumption $Z \subset D_{T}$ implies that $T \mid Z$ is bounded. Let $\left(x^{*}\right)^{\wedge}$ be the equivalence class of $x^{*} \in X^{*}$ in $X^{*} / Z^{\perp}$. Then, for every $x^{*} \in D_{T}$. and $x \in D_{U}$, one has

$$
\begin{aligned}
\left\langle T x, x^{*}\right\rangle & =\left\langle x, T^{*} x^{*}\right\rangle=\left\langle x,\left(T^{*} \mid Z^{\perp}\right)\left(x^{*}\right)^{\wedge}\right\rangle \\
& =\left\langle U x,\left(x^{*}\right)^{\wedge}\right\rangle=\left\langle U x, x^{*}\right\rangle .
\end{aligned}
$$

Since $D_{T^{*}}$ is total, (5.2) implies that $T x=U x$, for each $x \in D_{U}$. Since $T \mid Z$ is bounded and $U$ is a densely defined closed operator, it follows that $U=T \mid Z$ and hence $T^{*} / Z^{\perp}$ is the dual of $T \mid Z$.

Now we are in a position to prove our main theorem.

5.5. Theorem. Given $T \in C_{d}(X)$, the following assertion are equivalent:

(i) $T$ has the SDP;

(ii) for every pair of open disks $G, H$ with $\bar{G} \subset H$, there exist invariant subspaces $X_{G}$ and $X_{H}$ such that

$$
\begin{gathered}
X=X_{G}+X_{H} ; \quad X_{H} \subset D_{T} ; \\
\sigma\left(T \mid X_{H}\right) \subset H \quad \text { and } \quad \sigma\left(T \mid X_{G}\right) \subset G^{c} ;
\end{gathered}
$$

(iii) for every pair of open disks $G, H$ with $\bar{G} \subset H$, there exist invariant subspaces $Y, Z$ such that

(a) $\sigma(T \mid Y) \subset G^{c} ; T / Y$ is bounded and $\sigma(T / Y) \subset H$; 
(b) $Z \subset D_{T}, \sigma(T \mid Z) \subset H, T / Z$ is closed and $\sigma(T / Z) \subset G^{c}$;

(c) $T^{*} / Z^{\perp}$ is closed;

(iv) both $T$ and $T^{*}$ have property $(\beta)$;

(v) $T$ has property $(\beta)$ and $T^{*}$ has property $(\kappa)$.

Proof. The proof will be carried out through the following scheme of implications:

$$
\begin{aligned}
& \text { (i) } \Rightarrow \text { (ii) } \Rightarrow \text { (iv) } \quad \Rightarrow(\text { v }) \rightarrow(\text { i }) . \\
& \text { (i) } \Rightarrow \text { (iii) } \Rightarrow \text { (iv) }
\end{aligned}
$$

(i) $\Rightarrow$ (ii) is evident.

(i) $\Rightarrow$ (iii): Given $T$ with the SDP, let $G, H$ be open disks with $\bar{G} \subset H$ and let $L$ be an open set satisfying inclusions $\bar{G} \subset L \subset \bar{L} \subset H$. For $Y=X\left(T, G^{c}\right)$ and $Z=\overline{\Xi(T, L)}$, we have $X=Y+Z$. Then, in view of [3, Proposition 3.4 and Corollary 3.3], conditions (a) and (b) of (iii) are satisfied. Furthermore, it follows from (i) and [3, Theorem 9.8 (II,ii)], that

$$
Z^{\perp}=X^{*}\left(T^{*}, L^{c}\right) \text {. }
$$

Consequently, (iii,c) follows from [3, Proposition 3.4].

(ii) $\Rightarrow$ (iv): Let $G$ and $H$ be a pair of open disks with $\bar{G} \subset H$. There exists invariant subspaces $X_{G}$ and $X_{H}$ satisfying conditions (5.3) and (5.4). It follows from [3, Proposition 3.4] that $T / X_{G}$ is bounded and

$$
\sigma\left(T / X_{G}\right) \subset \sigma\left(T \mid X_{H}\right) \cup \sigma\left(T \mid X_{G} \cap X_{H}\right) \subset H .
$$

Then [3, Theorem 5.8] implies that $T$ has property $(\beta)$.

To show that $T^{*}$ has property $(\beta)$, let $\left\{f_{n}^{*}\right\}$ be a sequence of $D_{T^{*}}$-valued analytic functions defined on an open set $G \subset \mathrm{C}$ such that

$$
\left(\lambda-T^{*}\right) f_{n}^{*}(\lambda) \rightarrow 0 \quad(\text { as } n \rightarrow \infty)
$$

uniformly on every compact subset of $G$ in the strong topology of $X^{*}$. Without loss of generality, we may suppose that $G=\{\lambda:|\lambda|<r\}$ for some $r>0$ and that $K \subset G$ is compact. Let $G_{0}$ and $H_{0}$ be open disks satisfying inclusions

$$
K \subset G_{0} \subset \bar{G}_{0} \subset H_{0} \subset \bar{H}_{0} \subset G .
$$

Since $T$ has property $(\beta)$, the subspaces $X\left(T, G_{0}^{c}\right), \Xi\left(T, \bar{H}_{0}\right)$ are defined. In view of conditions (5.3) and (5.4) applied to the open disks $G_{0}, H_{0}$, one obtains

$$
X=X\left(T, G_{0}^{c}\right)+\Xi\left(T, \bar{H}_{0}\right) .
$$

Since $K \subset \rho\left(T \mid X\left(T, G_{0}^{c}\right)\right)$, for $\lambda \in K$ and $x \in X\left(T, G_{0}^{c}\right)$, one has $\left|\left\langle x, f_{n}^{*}(\lambda)\right\rangle\right|=\left|\left\langle R\left(\lambda ; T \mid X\left(T, G_{0}^{c}\right)\right) x,\left(\lambda-T^{*}\right) f_{n}^{*}(\lambda)\right\rangle\right| \leq M_{0}\left\|\left(\lambda-T^{*}\right) f_{n}^{*}(\lambda)\right\| \cdot\|x\|$, where $M_{0}>0$ is a constant independent of $\lambda \in K$. Then for every $\varepsilon>0$, there exists $N_{0}>0$ such that

$$
\left|\left\langle x, f_{n}^{*}(\lambda)\right\rangle\right| \leq \varepsilon\|x\|, \quad \text { for all } \lambda \in K \text { as } n>N_{0} .
$$


Let $C_{0}=\left\{\lambda:|\lambda|=r_{0}\right\} \subset G$ with $\bar{H}_{0}$ in the interior of the disk bounded by $C_{0}$, for some $r_{0}>0$. Then $C_{0} \subset \rho\left(T \mid \Xi\left(T, \bar{H}_{0}\right)\right)$ and hence for $\lambda \in C_{0}$ and $x \in \Xi\left(T, \bar{H}_{0}\right)$ one has

$$
\left|\left\langle x, f_{n}^{*}(\lambda)\right\rangle\right|=\left|\left\langle R\left(\lambda ; T \mid \Xi\left(T, \bar{H}_{0}\right)\right) x,\left(\lambda-T^{*}\right) f_{n}^{*}(\lambda)\right\rangle\right| \leq M_{1}\left\|\left(\lambda-T^{*}\right) f_{n}^{*}(\lambda)\right\|,
$$

where $M_{1}>0$ is a constant independent of $\lambda \in C_{0}$. Then there is $N_{1}$ such that

$$
\left|\left\langle x, f_{n}^{*}(\lambda)\right\rangle\right| \leq \varepsilon \frac{\operatorname{dist}\left(K, C_{0}\right)}{r_{0}}\|x\| \quad \text { for all } \lambda \in C_{0} \text { as } n>N_{1} .
$$

It follows from the Cauchy integral formula that

$$
\left|\left\langle x, f_{n}^{*}(\lambda)\right\rangle\right| \leq \frac{1}{2 \pi} \int_{|\xi|=r_{0}} \frac{\left|\left\langle x, f_{n}^{*}(\lambda)\right\rangle\right|}{|\xi-\lambda|}|d \xi| \leq \varepsilon\|x\|,
$$

for all $\lambda \in K$ as $n>N_{1}$.

The decomposition (5.5) and the inequalities (5.6), (5.7) imply that there is a constant $M>0$ such that

$$
\left|\left\langle x, f_{n}^{*}(\lambda)\right\rangle\right| \leq \varepsilon M\|x\| \text { for all } x \in X, \lambda \in K \text { as } n>\max \left\{N_{0}, N_{1}\right\} .
$$

Thus if follows that $\left\{f_{n}^{*}(\lambda)\right\}$ converges to zero uniformly on $K$ in the strong topology of $X^{*}$ and hence $T^{*}$ has property $(\beta)$.

(iii) $\Rightarrow$ (iv): Condition (iii,a) and [3, Theorem 5.8] imply that $T$ has property $(\beta)$. By Lemma 5.4, $Z^{*}=Z^{\perp}$ is invariant under $T^{*}$ and then

$$
\sigma\left(T^{*} \mid Z^{\perp}\right)=\sigma(T / Z) \subset G^{c} .
$$

Again, by Lemma 5.4, $T^{*} / Z^{\perp}$ is bounded and hence so is $T \mid Z$. We have

$$
\sigma\left(T^{*} / Z^{\perp}\right)=\sigma(T \mid Z) \subset H .
$$

Thus [3, Theorem 5.8] applies again and states that $T^{*}$ has property $(\beta)$.

(iv) $\Rightarrow$ (v) is evident.

(v) $\Rightarrow$ (i): Let $\left\{G_{0}, G_{1}\right\}$ be an open cover of $\mathbf{C}$, where $G_{0}$ is a neighborhood of infinity and $G_{1}$ is relatively compact. Let $U_{1}, U_{2}$ be a couple of Cauchy domains with $U_{1}$ bounded, $U_{2}$ unbounded such that $U_{2}=\left(\bar{U}_{1}\right)^{c}$. Furthermore, we request that $U_{2}$ verify inclusions

$$
G_{1}^{c} \subset U_{2} \subset \bar{U}_{2} \subset G_{0} .
$$

Next, we define the linear manifolds $N$ and $M$ as in $\S 4$. We claim that the following inclusions hold:

$$
\text { (a) } \quad N \subset \overline{X\left(T, G_{0}\right)}, \quad \text { (b) } \bar{M}^{w} \subset \Xi^{*}\left(T^{*}, \bar{G}_{1}\right) .
$$

To prove (5.8a), let $x \in N$. For $n=1,2,3, \ldots$ choose $f_{n} \in D_{H}$ such that $\left\|x-H f_{n}\right\|<1 / n$. Since $T$ has property $(\beta),\left\{f_{n}\right\}$ converges uniformly on compact sets in $U_{1}$. Put $f(\lambda)=\lim _{n \rightarrow \infty} f_{n}(\lambda)$, for $\lambda \in U_{1}$. Then $f(\lambda) \in D_{T}$ and $(\lambda-T) f(\lambda)=x, \lambda \in U_{1}$. Consequently,

$$
\sigma(x, T) \subset U_{1}^{c}=\bar{U}_{2} \subset G_{0}
$$

and $(5.8 a)$ follows. 
To prove (5.8b), let $x^{*} \in M$. There exists $g \in D_{H^{*}}$ such that $H^{*} g=0$ and $\tau g=x^{*}$, or equivalently,

$$
\left(\lambda-T^{*}\right) g(\lambda)=\tau g=x^{*}, \quad \lambda \in U_{2} .
$$

Thus it follows that

$$
\sigma\left(x^{*}, T^{*}\right) \subset U_{2}^{c} \subset \bar{G}_{1}
$$

and hence $x^{*} \in X^{*}\left(T^{*}, \bar{G}_{1}\right)$. Since $g(\lambda) \in V^{*}$ implies $\lim _{\lambda \rightarrow \infty}\|g(\lambda)\|=0$, it follows from [3, Lemma 5.11] that $x^{*} \in \Xi^{*}\left(T^{*}, \bar{G}_{1}\right)$. Therefore, $M \subset$ $\Xi^{*}\left(T^{*}, \bar{G}_{1}\right)$. Now [3, Theorem 9.4] implies that $\Xi^{*}\left(T^{*}, \bar{G}_{1}\right)$ is weak* closed and hence $\bar{M}^{w} \subset \Xi^{*}\left(T^{*}, \bar{G}_{1}\right)$. Now (5.8) and Theorem 4.2 imply

$$
\left(X\left(T, G_{0}\right)\right)^{\perp} \subset N^{\perp}=\bar{M}^{w} \subset \Xi^{*}\left(T^{*}, \bar{G}_{1}\right) .
$$

With $G_{0}$ fixed, we may choose a sequence of open sets $\left\{G_{n}\right\}$ such that $\bigcap_{n=1}^{\infty} \bar{G}_{n}=G_{0}^{c}=F_{0}$ and $\left\{G_{0}, G_{n}\right\}$ covers $\mathbf{C}$ for every $n$. Then (5.9) implies that

$$
\left(X\left(T, G_{0}\right)\right)^{\perp} \subset \Xi^{*}\left(T^{*}, \bar{G}_{n}\right) \text { for every } n .
$$

Consequently,

$$
\left(X\left(T, \bar{G}_{0}\right)\right)^{\perp} \subset \bigcap_{n=1}^{\infty} \Xi^{*}\left(T^{*}, \bar{G}_{n}\right)=\Xi^{*}\left(T^{*}, F_{0}\right) .
$$

Combining (5.10) with the evident inclusion $\left(X\left(T, G_{0}\right)\right)^{\perp} \supset \Xi^{*}\left(T^{*}, F_{0}\right)$, one finds

$$
\left(X\left(T, G_{0}\right)\right)^{\perp}=\Xi^{*}\left(T^{*}, F_{0}\right) .
$$

Since $\Xi^{*}\left(T^{*}, F_{0}\right)$ is invariant under $T^{*},(5.11)$ implies that $\overline{X\left(T, G_{0}\right)}$ is invariant under $T$. In fact, for every $x \in X\left(T, \bar{G}_{0}\right) \cap D_{T}$ and $x^{*} \in \Xi^{*}\left(T^{*}, F_{0}\right)$, one has $\left\langle T x, x^{*}\right\rangle=\left\langle x, T^{*} x^{*}\right\rangle=0$ so that $\overline{X\left(T, G_{0}\right)}$ is invariant under $T$. Furthermore, we shall show that

$$
\sigma\left(T \mid \overline{X\left(T, G_{0}\right)}\right) \subset \bar{G}_{0} .
$$

Let $x \in \overline{X\left(T, G_{0}\right)}$ and choose a sequence $\left\{x_{n}\right\} \subset X\left(T, G_{0}\right)$ such that $x_{n} \rightarrow$ $x$. Let $x_{n}(\cdot)$ denote the local resolvent of $T$ at $x_{n}$. By property $(\beta)$, the convergence

$$
(\lambda-T) x_{n}(\lambda)=x_{n} \rightarrow x, \quad \lambda \in\left(\bar{G}_{0}\right)^{c}
$$

implies $x_{n}(\lambda) \rightarrow f(\lambda)$ and $(\lambda-T) f(\lambda)=x$.

Therefore $\sigma(x, T) \subset \bar{G}_{0}$. On the other hand, for every $\lambda \in\left(\bar{G}_{0}\right)^{c}$, we have

$$
\sigma\left(x_{n}(\lambda), T\right)=\sigma\left(x_{n}, T\right) \subset G_{0},
$$

so $x_{n}(\lambda) \in X\left(T, G_{0}\right)$ and hence $x(\lambda) \in \overline{X\left(T, G_{0}\right)}$ for $\lambda \in\left(\bar{G}_{0}\right)^{c}$. Then, by a known property [5, see also 3, Proposition 2.7], inclusion (5.12) follows.

Now we are in a position to show that $T$ has the SDP. Let $\left\{G_{0}, G_{1}\right\}$ be an open cover of $\mathbf{C}$ with $G_{0}$ a neighborhood of infinity and $G_{1}$ relatively compact. 
Let $H_{0}$ be another open neighborhood of infinity such that $\bar{G}_{1} \cap \bar{H}_{0}=\varnothing$ and $\bar{H}_{0} \subset G_{0}$. Then $\widetilde{G}_{0}=G_{1} \cup H_{0}$ is a neighborhood of infinity and in virtue of (5.11) one writes

$$
X\left(T, \widetilde{G}_{0}\right)^{\perp}=\Xi^{*}\left(T^{*}, \widetilde{F}_{0}\right),
$$

where $\widetilde{F}_{0}=\left(\widetilde{G}_{0}\right)^{c}$ and both $\Xi^{*}\left(T^{*}, F_{0}\right), \Xi^{*}\left(T^{*}, \widetilde{F}_{0}\right)$ are closed in the weak* topology of $X^{*}$. Similarly, $\Xi^{*}\left(T^{*}, F_{0} \cup \widetilde{F}_{0}\right)$ is closed in the weak* topology. Since $F_{0} \cap \widetilde{F}_{0}=\varnothing \quad\left(F_{0}=G_{0}^{c}\right)$, we have

$$
\Xi^{*}\left(T^{*}, F_{0} \cup \widetilde{F}_{0}\right)=\Xi^{*}\left(T^{*}, F_{0}\right) \oplus \Xi^{*}\left(T^{*}, \widetilde{F}_{0}\right) .
$$

Set $Z^{*}=\Xi^{*}\left(T^{*}, F_{0} \cup \widetilde{F}_{0}\right)$.

Let $x \in X, x^{*} \in Z^{*}$ and denote by $x_{0}^{*}$ the projection of $x^{*}$ onto $\Xi^{*}\left(T^{*}, F_{0}\right)$, in conjunction with (5.13). The linear functional $x_{0}$ on $Z^{*}$, defined by

$$
\left\langle x_{0}, x^{*}\right\rangle=\left\langle x, x_{0}^{*}\right\rangle
$$

is continuous in the weak* topology. Use the Hahn-Banach theorem on locally convex spaces to extend $x_{0}$ to a linear functional on $X^{*}$, that is continuous in the weak* topology. Therefore $x_{0} \in X$. Since the projection $x_{0}^{*}$ of $x^{*} \in$ $\Xi^{*}\left(T^{*}, \widetilde{F}_{0}\right)$ onto $\Xi^{*}\left(T^{*}, F_{0}\right)$ is zero, it follows from $(5.14)$ that $\left\langle x_{0}, x^{*}\right\rangle=$ 0 for $x^{*} \in \Xi^{*}\left(T^{*}, \widetilde{F}_{0}\right)$. Thus, $x_{0} \in{ }^{\perp} \Xi^{*}\left(T^{*}, \widetilde{F}_{0}\right)=\overline{\left(X\left(T, \widetilde{G}_{0}\right)\right.}$. Put $x_{1}=$ $x-x_{0}$ and for $x^{*} \in \Xi^{*}\left(T^{*}, F_{0}\right)$, use (5.14) to obtain $\left\langle x_{1}, x^{*}\right\rangle=0$. Then $x_{1} \in{ }^{\perp} \Xi^{*}\left(T^{*}, F_{0}\right)=\overline{X\left(T, G_{0}\right)}$. Since $x \in X$ is arbitrary, the representation $x=x_{0}+x_{1}$ with $x_{0} \in \overline{X\left(T, \widetilde{G}_{0}\right)}, x_{1} \in \overline{X\left(T, G_{0}\right)}$ implies

$$
X=\overline{X\left(T, G_{0}\right)}+\overline{X\left(T, \widetilde{G}_{0}\right)}
$$

As regarding $\overline{X\left(T, \widetilde{G}_{0}\right)}$, it follows from (5.12) that

$$
\sigma\left(T \overline{X\left(T, \widetilde{G}_{0}\right)}\right) \subset \overline{\widetilde{G}}_{0}=\bar{G}_{1} \cup \bar{H}_{0} .
$$

Having $\bar{G}_{1} \cap \bar{H}_{0}=\varnothing$ and $G_{1}$ relatively compact, the functional calculus for closed operators produces the following decomposition

$$
\begin{array}{ll}
\text { (a) } \overline{X\left(T, \widetilde{G}_{0}\right)}=Y_{1} \oplus Y_{2}, & \text { (b) } Y_{1} \subset D_{T} \\
\text { (a) } \sigma\left(T \mid Y_{1}\right) \subset \bar{G}_{1}, & \text { (b) } \sigma\left(T \mid Y_{2}\right) \subset \bar{H}_{0} .
\end{array}
$$

Since $\bar{H}_{0} \subset G_{0}, Y_{2} \subset \overline{X\left(T, G_{0}\right)},(5.15)$ and (5.16) imply

$$
X=Y_{1}+\overline{X\left(T, G_{0}\right)} \text {. }
$$

In view of (5.16b), (5.12), (5.17a) and (5.18), $T$ has the SDP.

Remark. A more restrictive version of property $(\beta)$ is used in [6, Lemma 4.6]. Given $T \in C(X)$, a function $f: G \rightarrow D_{T}$ defined on an open subset $G$ of the compactified complex plane $\mathbf{C}_{\infty}$, is said to be $T$-analytic if both $f$ and $T f$ are 
analytic on $G . T$ has property $(\beta)$, in this stronger version, if for any sequence of $T$-analytic functions $\left\{f_{n}: G \rightarrow D_{T}\right\}$, the condition $(\lambda-T) f_{n}(\lambda) \rightarrow 0$ (as $n \rightarrow \infty)$ in the strong topology of $X$ and uniformly on every compact subset of $G$ implies that $f_{n}(\lambda) \rightarrow 0$ in the strong topology of $X$ and uniformly on every compact subset of $G$.

It follows from the definition of the operator $H$ and Lemma 2.2 in $\S 2$ that both $T f(\mu)$ and $T^{*} g(\lambda)$ are analytic. Consequently, Theorem 5.5 holds if we use the above-mentioned stronger version of property $(\beta)$ in (iv) and (v).

\section{REFERENCES}

1. E. Bishop, A duality theorem for an arbitrary operator, Pacific J. Math. 9 (1959), 379-397.

2. N. Dunford and J. T. Schwartz, Linear operators, Part III, Wiley, New York, 1971.

3. I. Erdelyi and Wang Shengwang, A local spectral theory for closed operators, London Math. Soc. Lecture Note Ser. 105, Cambridge Univ. Press, 1985.

4. R. Lange and Wang Shengwang, New criteria for a decomposable operator, Illinois J. Math. (in print).

5. R. C. Sine, Spectral decomposition of a class of operators, Pacific J. Math. 14 (1964), 333-352.

6. Florian-Horia Vasilescu, Analytic functional calculus and spectral decompositions, Reidel, Dordrecht, Boston and London 1982.

Department of Mathematics, Temple University, Philadelphia, Pennsylvania 19122

Department of Mathematics and Statistics, University of Pittsburgh, Pittsburgh, Pennsylvania 15260 